\title{
Chronische Schmerzen in Deutschland: Prävalenz und sozioökonomische Relevanz
}

\author{
Wie häufig sind chronische \\ Schmerzen in der deutschen \\ Bevölkerung und welche sozio- \\ könomischen Auswirkungen \\ haben sie?
}

A m 5. Juni 2012 führte die Deutsche Schmerzgesellschaft den 1. Nationalen Aktionstag gegen den Schmerz durch. Ziel ist es, die Versorgung von Patienten mit chronischen Schmerzen zu verbessern. In ihrer Pressemitteilung gab die Deutsche Schmerzgesellschaft an, dass $17 \%$ der Deutschen an nicht tumorbedingten chronischen Schmerzen leiden und von diesen bis zu $50 \%$ eine unzureichende Schmerztherapie erhalten. Die Studie wurde 2007 deutschlandweit durchgeführt.

Die Befragung erfolgte mithilfe des Sleep-EVAL Knowledge-Based Expert Systems. Erfasst wurden das Vorliegen chronischer Schmerzen (definiert als über drei Monate an mindestens drei Tage in der Woche anhaltende Schmerzen), Schmerzlokalisationen, schmerzmodulierenden Faktoren, Einfluss der Schmerzen auf Alltagsaktivitäten, die Lebensqualität, körperliche Erkrankungen und seelische Störungen sowie Behandlungen aufgrund von Schmerzen. Zur Erfassung neuropathischer
Schmerzen wurden die validierten Instrumente DN4Q („Douleur Neuropathique en 4 questions") und painDETECT eingesetzt.

Von 3.011 Studienteilnehmern berichteten $24,9 \%$ über chronische Schmerzen. Bei 6,5\% lag ein chronisches Schmerzsyndrom mit neuropathischen Komponenten vor. Die Teilnehmer wurden nach dem Vorliegen von neuropathischen Schmerzkomponenten differenziert. Berichtet wurden die Raten von Schmerzsyndromen mit und ohne neuropathische Schmerzkomponenten bei Teilnehmern mit Majorer Depression $(30,7$ vs. $20,2 \%)$ und mit Angststörung (26,8 vs. $11,7 \%)$.

Fazit: Die Autoren folgern, dass Menschen mit chronischen Schmerzen mit neuropathischen Komponenten stärker beeinträchtigt sind als solche ohne neuropathische Komponenten.

Ohayon MM, Stingl JC. Prevalence and comorbidity of chronic pain in the German general population. J Psychiatr Res. 2012;46(4):444-50.

Kommentar von Winfried Häuser, Saarbrücken: Die Studie hat einige Limitationen: Zur Erfassung von Beeinträchtigungen und Lebensqualität wurden keine validierten
Fragebögen verwendet. Krebserkrankungen als mögliche Ursachen chronischer Schmerzen wurden nicht erfasst. Die Teilnehmer mit chronischen Schmerzen wurden nicht mit alters- und geschlechtsgematchten Teilnehmern ohne chronische Schmerzen verglichen. Die Bedeutung neuropathischer Schmerzen wurde überschätzt, weil mit den verwendeten Instrumenten neuropathische Schmerzkomponenten auch bei Krankheitsbildern identifiziert werden, welche üblicherweise nicht als solche angesehen werden, zum Beispiel das Fibromyalgiesyndrom.

Dennoch erweitert die Studie den Kenntnisstand zur Häufigkeit und sozioökonomischen Konsequenzen chronischer Schmerzen in Deutschland und unterstützt die von der Deutschen Schmerzgesellschaft genannten Zahlen. Auch zeigt sie, dass sich nicht alle Menschen mit chronischen Schmerzen beeinträchtigt fühlen und/oder medizinische Behandlung in Anspruch nehmen. Nicht alle Menschen mit chronischen Schmerzen sind "Schmerzpatienten" und nicht alle "Schmerzpatienten" sind "schmerzkrank" (chronischer Schmerz mit negativen biopsychosozialen Konsequenzen).

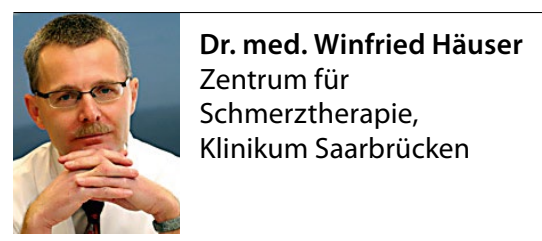

\section{Optimistische Menschen halten Schmerzen scheinbar besser aus}

\section{Positiv gestimmte Menschen empfinden Schmerzen nicht so stark wie Schwarzseher. Dies hat eine niederländische Studie mit 79 Studenten gezeigt.}

U m deren Optimismus zu objektivieren, hatten die Autoren experimentell für verschiedene Stimmungslagen gesorgt: Die eine Hälfte der Teilnehmer durfte gedanklich in möglichst angenehmen Visionen für die eigene Zukunft schwelgen (Best Possible Self, BPS), die anderen sollten sich ein nüchternes Szenario vor Augen führen (Typical Day, TD). Die BPS-Manipulation sorgte nach- weislich für mehr Optimismus, dies konnten die Forscher mit dem „Future Expectations“-Fragebogen feststellen. Alle Teilnehmer wurden sodann aufgefordert, die Hand für eine Minute in ein $5{ }^{\circ} \mathrm{C}$ kaltes Wasserbad zu tauchen.

Ergebnis: Auf einer Skala von 0 (kein Schmerz) bis 10 (extremer Schmerz) gaben die positiv "gepolten“ Studenten signifikant niedrigere Werte an als die Vergleichsgruppe. Die Autoren postulieren einen ursächlichen Zusammenhang, der über zwei Mechanismen läuft: einerseits die Schmerzerwartung, andererseits das sogenannte „Katastrophisieren“. Im Experiment hatten diejenigen die heftigste Pein empfunden, die bereits im Vorfeld mit starken Schmerzen gerechnet hatten und generell dazu neigten, Schmerzen als bedrohlich zu empfinden.

Fazit: Die Studie legt zweierlei nahe: Erstens, dass sich Schmerzen durch Interventionen, die positives Denken fördern, lindern lassen, zweitens, dass man Optimismus durchaus lernen kann.

Elke Oberhofer

Hanssen MM et al. Optimism lowers pain:

Evidence of the causal status and underlying mechanisms. Pain. 2013;154(1):53-8. 